\title{
ESTUDIO COMPARATIVO DE LA COMPOSICIÓN BIOMINERAL DE CUATRO ESPECIES DEL GÉNERO GUNNERA DE LA ARgeNTINA
}

\author{
YAMILA C. ACOSTA RICCII', MARÍA G. FERNÁNDEZ PEPI² y MIRTA O. ARRIAGA ${ }^{3}$
}

\begin{abstract}
Resumen: Este trabajo tuvo por objeto caracterizar y analizar, comparativamente, la producción de biominerales en especies del género Gunnera presentes en Argentina (G. apiculata, G. lobata, G. magellanica y $G$. tinctoria). Se describe y analiza cualitativa y cuantitativamente los biominerales tanto cálcicos como silíceos, ubicados en sus tejidos. Se ha identificado elementos anatómicos foliares con validez taxonómica que permiten diferenciar los taxa a nivel de especie o grupo de especies, como ser, la forma de los extremos de los ejes, tamaño y número de los calciofitolitos; morfotipos silíceos predominantes. Los resultados obtenidos en el estudio de las biomineralizaciones pueden relacionarse con las características del hábitat donde crecen estas plantas, pudiendo ser útiles como herramientas para el estudio ambiental y/o de vegetaciones pasadas de esas regiones.
\end{abstract}

Palabras clave: Silicofitolito, calciofitolito, Gunnera, Argentina.

Summary: Comparative study of the bio-mineral composition of four species of the genus Gunnera in Argentina. This study aimed to characterize and analyze comparatively, the biominerals of species of the genus Gunnera from Argentina (G. apiculata, G. lobata, G. magellanica and G. tinctoria). It is described and analyzed both qualitatively and quantitatively calcium biominerals as siliceous, located in the leaf tissues. We identified taxonomic validity anatomical elements that distinguish the taxa to species or group of species, such as the shape of the ends axis, the size and number the dominant calcium and silica phytoliths morphotypes. The results obtained in the study of biomineralizations may relate to the characteristics of the habitat where plants grow and may be useful as tools for environmental assessment and/or past vegetation in these regions.

Key words: Silicophytoliths, calcium phytolith, Gunnera, Argentina.

\section{INTRODUCCIÓN}

La familia Gunneraceae Meisn. comprende únicamente al género Gunnera L., el cual está conformado por plantas herbáceas perennes, de tamaño variable, desde pequeñas hasta gigantes, monoicas o dioicas. Presentan rizoma rastrero, estolonífero con o sin catáfilos y hojas pecioladas,

\footnotetext{
${ }^{1}$ Laboratorio de Anatomía Vegetal, Museo Argentino de Ciencias Naturales Bernardino Rivadavia MACN CONICET, Av. Ángel Gallardo 470 (C1405DJR), yamilaricci@gmail.com

${ }^{2}$ Cátedra de Nutrición Animal, Facultad de Agronomía FAUBA, Av. S. Martín 4453 (C1417DSE), fernandezpepi@ agro.uba.ar.

${ }^{3}$ Laboratorio de Anatomía Vegetal, Museo Argentino de Ciencias Naturales Bernardino Rivadavia MACN CONICET, Av. Ángel Gallardo 470 (C1405DJR), mirtaarriaga@gmail.com
}

enteras, lobadas, crenadas o serradas, con nervios muy marcados en la cara abaxial (Molina, 1978; Wilkinson, 2000). Las hojas Frecuentemente están recubiertas por pelos, verrugas y emergencias; algunos de esos pelos pueden llegar a ser glandulares. Dichas estructuras glandulares en la zona del tallo, pueden servir de entrada a colonias de algas azules del género Nostoc (Metcalfe \& Chalk, 1950; Wilkinson, 2000). Los pelos presentes en especies del género Gunnera pueden ser unicelulares simples o pluricelulares glandulares (Metcalfe \& Chalk, 1950; Napp-Zinn, 1984). La variedad morfológica de los pelos dentro de las especies del género, es útil como carácter taxonómico, especialmente en lo referente a su forma, ubicación y contenidos celulares. La mayor parte de las especies de este género se encuentran en Sudamérica. En Argentina, es posible hallar cinco especies: G. apiculata Schindl., G. lobata Hook. f., G. magellanica Lam., 
G. schindleri L. E. Mora y G. tinctoria (Mol.) Mirbel (Zuloaga \& Morrone, 1999; Zuloaga et al. 2008). En el territorio que abarcan las provincias de Neuquén hasta Santa Cruz, Tierra del Fuego e Islas Malvinas habitan G. tinctoria y G. magellanica; $G$. lobata se restringe a la provincia de Tierra del Fuego (Molina, 1978), mientras que G. schindleri y $G$. apiculata lo hacen en las provincias de Jujuy y Salta respectivamente.

De G. schlindleri solo se conoce su ejemplar tipo, por lo cual no pudo ser incluida en este estudio, dado que no se le pudo aplicar los tratamientos desarrollados en este trabajo.

En los vegetales es muy común la formación de biomineralizaciones o fitolitos. Estos depósitos pueden ser de sílice amorfa (silicofitolitos) o de oxalato de calcio (calciofitolitos) (Bertoldi de Pomar, 1975). El sílice es uno de los constituyentes inorgánico más importante en las plantas superiores, ya que está involucrado en funciones fisiológicas que son de vital importancia para la planta, entre ellas: crecimiento, desarrollo, viabilidad, reproducción, toxicidad por metales pesados, etc. (Aguirre et al. 2007). Dicho mineral, se encuentra ampliamente distribuido en el cuerpo vegetal y por su riqueza, una vez muerta la planta, es imposible eliminarlo de los sustratos en donde ellas crecen (Aguirre et al., 2007). Dichas características le confiere a la sílice, un papel protagónico en estudios paleoambientales y paleobotánicos. Constituyendo una herramienta importante en sí mismo y en especial, cuando es complementado con otros estudios, tales como caracterización de polen y esporas (Bertoldi de Pomar, 1975).

Debido a que los silicofitolitos son estables en su naturaleza química, pueden perdurar en los suelos por mucho tiempo, con lo cual pasan a formar parte de los materiales sedimentarios asociados a las plantas que les dieron origen (Bertoldi de Pomar, 1975; BorbaRoschel et al., 2006). Estos cuerpos de sílice amorfo se acumulan progresivamente, en los horizontes superiores, en cantidades variables, dependiendo de la población vegetal que dicho suelo soporte, lo que demuestra el valor taxonómico de los silicofitolitos como herramienta en los campos de la Botánica, Paleobotánica, Paleoecología y Arqueología (Bertoldi de Pomar, 1975; Fernández Pepi et al., 2012a). Analizando los morfotipos silíceos propios de cada especie, pueden establecerse patrones de identificación generales y específicos. Con esta determinación se logra no solo precisar la vegetación actual, sino también la secuencia a través del tiempo, pudiendo establecer los cambios composicionales o climáticos del área en estudio (Bertoldi de Pomar, 1975; Pérez Cuadra \& Hermann, 2013; Fernández Pepi et al., 2012b). El origen, la distribución y la morfología de las biomineralizaciones de origen cálcico o calciofitolitos, en las plantas superiores, responde a patrones específicos (Webb, 1999). Franceschi \& Nakata (2005) mencionan como razones fundamentales de dicha variabilidad a los factores genéticos y ambientales. Los cristales suelen ser de oxalato de calcio, producto de la unión del ácido oxálico sintetizado endógenamente y el calcio del medio en el que se desarrolla la planta (Franceschi \& Nakata, 2005). Este complejo tiene variables morfologías y se ubica en todos los órganos de la planta ya sea, raíz, tallo, hoja, flores, frutos y semillas, dentro de tejidos epidérmicos, vasculares y parenquimático, formados en células especializadas llamadas idioblastos. Dichas células acumulan materiales y difieren del resto de las células que componen los tejidos (Webb, 1999; Franceschi \& Nakata, 2005). Se describieron variedades de cristales de oxalato de calcio; (Webb, 1999; Franceschi \& Nakata, 2005; Pérez Cuadra \& Hermann, 2013) que permiten distinguir a los grupos de plantas entre sí, generalmente, en la morfología y ubicación de dichos cristales. Pero a pesar del gran rango de variación, las características mencionadas anteriormente se mantienen constantes entre individuos de una misma especie. Siendo ésta una característica taxonómica importante al momento de la distinción interespecífica de individuos, y a su vez, en el agrupamiento de otros (Webb, 1999; Lersten \& Horner, 2009). El objetivo de este trabajo fue realizar un análisis descriptivo-comparativo de las biomineralizaciones presentes en las hojas de las cuatro especies de Gunnera de la Argentina, a fin de establecer caracteres diagnósticos de las mismas.

\section{Materiales y Métodos}

\section{Materiales estudiados}

Para este estudio se utilizaron cuatro de las cinco especies del género Gunnera que se distribuyen en Argentina (Fig. 1). Se trabajó con muestras de hojas de ejemplares provenientes de los siguientes Herbarios: del Museo Argentino de Ciencias 
Naturales (BA), del Instituto de Botánica Darwinion (SI) y del Museo de Ciencias Naturales de La Plata (LP). Todos los materiales son de Argentina, salvo indicación contraria.

G. apiculata: Prov. Jujuy: Dpto. Capital, Lagunas de Yala, Cabrera \& Kiesling 20141, 4/ XII/1969 (LP). Dpto. Tilcara, Yala de Monte
Carmelo, Fabris et al. 6512, 19-21/I/1966 (LP). Dpto. Valle Grande, Serranía de Calilegua, Fabri, et al. 5761, 15-17/XI/1965 (LP). Prov. Neuquén: S/ Dpto., en torrentes, Cabrera 11247, 14/XII/1952 (LP). Prov. Río Negro: Dpto. Bariloche, San Carlos de Bariloche; Burkart 6110, 28/I/1934 (BA). Prov. Salta: Dpto. Santa Victoria, Lizoite, Crespo 37090, 24/XI/1971(BA).

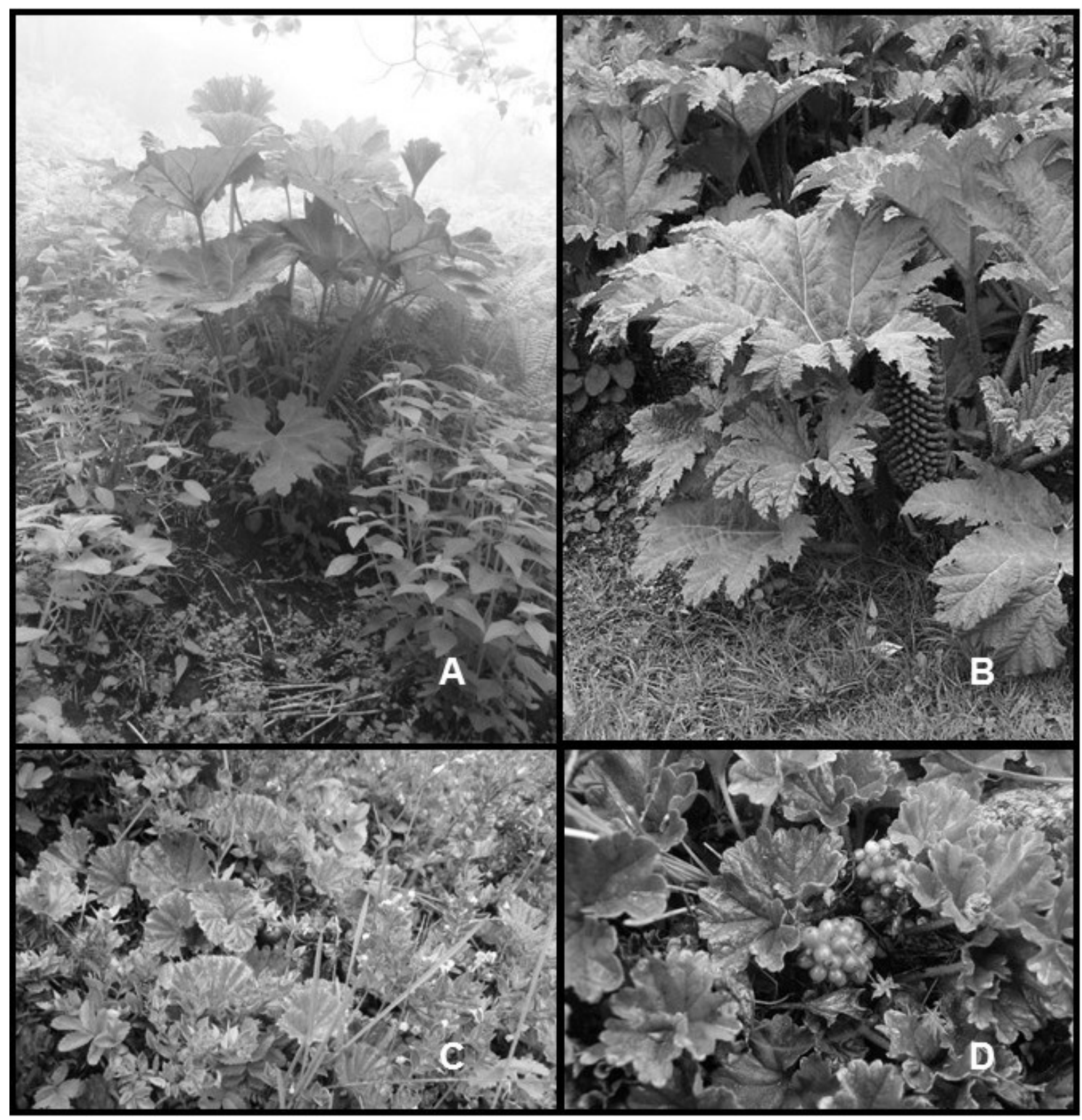

Fig. 1. A. Gunnera apiculata. B: Gunnera tinctoria. C: Gunnera magellanica. D: Gunnera lobata. 
G. lobata: Prov. Santa Cruz: Dpto. Lago Argentino, Camino a la Laguna Fría; Cabrera et al. 25895, 16/XI/1975 (LP). Isla de los Estados: Puerto San Juan, Castellanos 12941, 31/XII/1933 (BA).

G. magellanica: Prov. Neuquén: Dpto. Los Lagos, Lago Nahuel Huapi, brazo del Machete, Pérez Moreau 35331, II/1940 (BA). Lago Fonck, Pérez Moreau 49404, 21/I/1942 (BA). Prov. Río Negro: Dpto. Bariloche, Lago Frías, Pérez Moreau 35334 BA, 17/I/1940. Cerro Righi, Pérez Moreau 35335, 23/I/1940 (BA). Prov. Tierra del Fuego e Islas de los Estados: Dpto. Agua Fresca, sur de Punta Arenas, Molina.65015, 26/VI/1977 (BA). Dpto. Arroyo Beta, Castellanos 45696, 20/II/1942 (BA). Dpto. Bahía Crosley, Correa 83683, 10/ III/1962 (BA). Dpto Ushuaia, Bahía Lapataia, Pérez Moreau \& Guarrera 44353, 1-4/II/1948 (BA). Ibidem, Lago Roca, Correa et al. 83682, XII/1912 (BA).Dpto. Isla de los Estados, Puerto San Juan, Castellanos 12941, 31/XII/1933 (BA). CHILE: Prov. de Magallanes: Río Las Minas, Donat 8188, 26/VI/1977 (BA).

G. tinctoria: Prov. Chubut: Dpto. Cushamen, Lago Puelo, Pérez Moreau 45329, 18/I/1941 (BA). Prov. Neuquén: Dpto. Los Lagos, Puerto Blest, Arroyo Bravo; Castellanos 22031, 9/II/1934 (BA). S/Dpto., en Torrentes, Cabrera 11247, 14/XII/1952 (LP). Prov. Río Negro: Dpto. Bariloche, Lago Frías, Pérez Moreau 49405, 01/1942 (BA); Ibidem, Manso inferior, Dimitri y Correa 86433, II/1961 (BA). Ibidem, Bariloche (material cultivado en jardín), Ezcurra 93065, III/2013 (BA). Ibidem, Quebradas Húmedas, Burkart 12298, 28/I/1934 (BA). Prov. Tucumán: Dpto. Trancas, Tapia, Venturi 1046, 14XI-1920 (BAA, LIL). CHILE. Dpto. Llanquihue, Petrohué; Perez Moreau 23257, III/1938 (BA).

\section{Obtención de las biomineralizaciones}

El material foliar, de cada ejemplar de las cuatro especies incluidas en este estudio, se lavó con detergente neutro y luego con agua destilada. Se secó a estufa $\left(60^{\circ} \mathrm{C}\right)$ y se lo pesó para obtener el peso de materia seca (peso seco). Para la obtención de fitolitos se siguió la técnica de Labouriau (1983), se sometieron las muestras a una carbonización a $200^{\circ} \mathrm{C}$ durante dos horas. Al material obtenido se le realizó una digestión química con $\mathrm{HCl} 5 \mathrm{~N}$. Se realizaron lavados con agua destilada en papel de filtro libre de cenizas, hasta lograr liberar a las muestras de cloruros. Se sometió al material a una calcinación a $800^{\circ} \mathrm{C}$, durante dos horas. Las cenizas obtenidas del proceso fueron pesadas.

Otras submuestras fueron tratadas con la técnica de Labouriau (1983), sin efectuar el tratamiento con $\mathrm{HCl}$, de manera de obtener tanto el material silíceo como cálcico. Para cada tratamiento, en cada especie, se calculó el índice de biomineralización total, silícea y cálcica, que relaciona el contenido del tipo de biomineralización en función de la materia seca inicial, respectivamente, siguiendo la siguiente fórmula (Fernández Honaine et al., 2005; Fernández Pepi et al., 2012a; Dey et al., 2015):
$\mathrm{IB}=$ masa de cenizas obtenidas $(\mathrm{g}) \times 100$ masa inicial $(\mathrm{g})$

Los índices obtenidos de las muestras por ambos tratamientos se compararon a fin de poder establecer una relación entre la proporción de los tipos de biomineralizaciones respecto a las totales.

Las cenizas obtenidas fueron montadas en aceite de inmersión para su observación microscópica, realizándose la identificación y cuantificación relativa de las formas observadas. Para ello se utilizó un microscopio óptico (MO) Leica DMLB. La identificación de las biomineralizaciones cristalinas (cálcicas) y amorfas (silíceas) se realizó mediante la observación de los preparados microscópicos de cenizas, epidermis y cortes transversales bajo luz polarizada. Se han realizado 65 preparados de cenizas de las cuatro especies. Los preparados fueron observados con MO y fotomicrografiados, identificándose $\mathrm{y}$ describiéndose las biomineralizaciones halladas. Luego de describir su morfología se tomaron datos cuantitativos.

Clasificación y descripción de los morfotipos de sílice amorfo

La clasificación se realizó siguiendo la terminología propuesta por Zucol et al. (2010), Patterer et al. (2011), Fernández Pepi et al. (2012a) y Fernández Pepi (2013). La cuantificación de los silicofitolitos se realizó en cada preparado de cenizas, tomando en cuenta la frecuencia de aparición de cada morfotipo. Se realizaron 16 preparados totales por especie. 
Clasificación y descripción de elementos cálcicos

Por el método de diafonización descripto por D’ Ambrogio de Argüeso (1986), se prepararon las epidermis foliares, para la observación y descripción de biominerales cálcicos. A éstos últimos se los clasificó sensu Pérez Cuadra \& Hermann (2013), por los contornos delimitados por los extremos de los cristales que componen las drusas. Se realizó el recuento de las drusas presentes por campo microscópico, con aumentos de 100x, en preparados epidérmicos. Se contaron 10 campos para cada especie. Según los resultados se los clasificó en las siguientes categorías: escaso (cuando se observan hasta 20 drusas por campo); abundante (entre 20 y 60 drusas por campo); y muy abundante (más de 60 drusas por campo).

\section{Análisis de datos}

Se realizó la comparación entre las especies estudiadas, teniendo en cuenta los contenidos de las biomineralizaciones totales, silíceas y cálcicas, mediante test no paramétrico de KruskalWallis. Además se utilizaron técnicas de análisis multivariado (Análisis de correspondencia) para diferenciar a las especies según las asociaciones de los silicofitolitos presentes en cada una. Dichos análisis se realizaron mediante el programa estadístico Past software (Hammer et. al. 2007).

\section{Resultados}

\section{Índice de biomineralización}

A partir de las cenizas obtenidas por los tratamientos con y sin $\mathrm{HCl}$ se calculó, para cada especie, el índice de biomineralización total, el silíceo y el cálcico (Fig. 2). Al comparar los índices de biomineralizaciones totales, G. lobata presenta diferencias significativas con el resto de las especies, siendo la de menor índice $(\mathrm{p}=0,003)$ (Fig. 2). Teniendo en cuenta el origen de las biomineralizaciones, $G$ lobata y $G$. apiculata no presentan diferencias significativas entre los índices de biomineralización silícea y cálcica $(\mathrm{p}=0,007)$. Sin embargo, en $G$. magellanica y $G$. tinctoria sí se observan diferencias significativas entre dichos índices, siendo mayor la proporción de biominerales cálcicos ( $\mathrm{p}=0,002)$ (Fig. 2). Los porcentajes de los contenidos silíceos en cada especie presentan diferencias significativas entre
G. apiculata y G. tinctoria con respecto a las otras dos especies $(\mathrm{p}<0,005)$, siendo mayor en las dos primeras (Fig. 2).

\section{Descripción de las asociaciones fitolíticas}

Calciofitolitos: En los cortes epidérmicos, se identifica la presencia de calciofitolitos de tipo drusas, las cuales están formadas por la concreción de numerosos cristales prismáticos individuales, presentes en las células del parénquima clorofiliano de hoja y pecíolo de todas las especies. Según el contorno de las drusas estas han sido clasificadas en: Drusas con extremos de las proyecciones redondeados, los cristales que componen la drusa tienen extremos redondeados, observadas en $G$. lobata (Fig. 3A); Drusas con extremos truncos, los cristales tienen extremos truncados, presentes en G. magellanica (Fig. 3C); Drusas con extremos agudos, los cristales presentan extremos agudos, como punta de flecha, encontradas en G. apiculata y G. tinctoria (Fig. 3B, D). Se ha observado que la abundancia de las drusas varía según la especie, siendo muy abundantes en $G$. tinctoria donde se han contabilizado un máximo de 200 drusas por campo microscópico. En G. magellanica y $G$. apiculata estas son abundantes presentando un máximo de 45 y 51 drusas por campo microscópico respectivamente, y escasas en $G$. lobata donde se encuentra un máximo de 20 drusas por campo (Tabla 1). También se observó variación interespecífica en el tamaño de las drusas, siendo las de G. tinctoria las drusas con mayor tamaño y las de G. magellanica las de menor tamaño (Tabla 2). Algunas drusas se observaron envueltas en una membrana (membrana de Rosanoff), que le sirve de protección como en el caso de G. apiculata (Fig. 3B).

Silicofitolitos: Se identificaron morfotipos no articulados (Fig. 4), como así también articulados (Fig. 5). Dentro de los no articulados, encontramos elementos irregulares (Ir), derivados de células epidérmicas de bordes ondulados, los elementos poliédricos $(\mathrm{Pe})$ de bordes lisos y los derivados de pelos unicelulares y uniseriados (Dp) (Fig. 4). Dentro de los morfotipos articulados, con elementos derivados de epidermis con diversos grados de silicificacion y articulación, que incluyen células epidérmicas y bases de pelos (Fig. 5). 
Bol. Soc. Argent. Bot. 50 (4) 2015

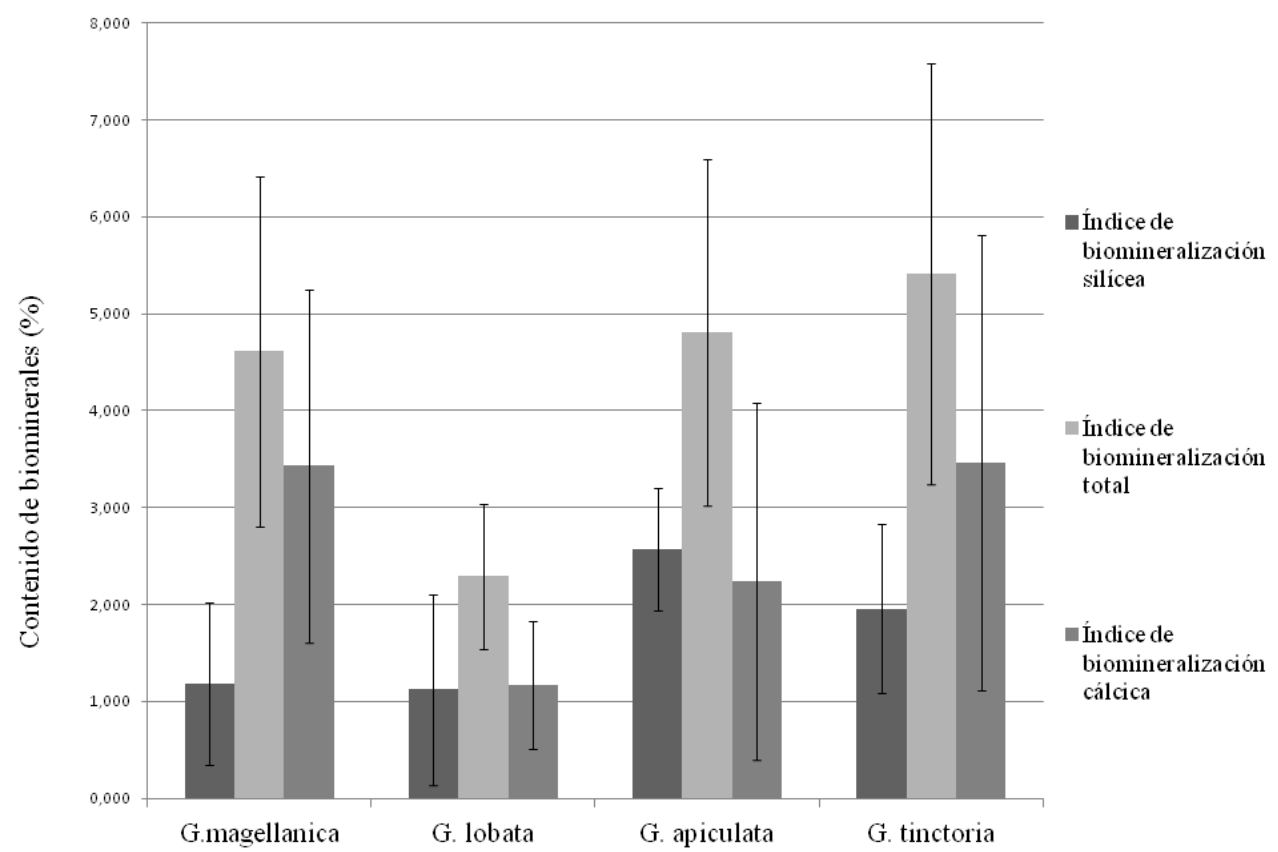

Fig. 2. Contenido de biominerales totales, de origen silíceo y cálcico, para G. magellanica, G. lobata, G. apiculata y $G$. tinctoria.
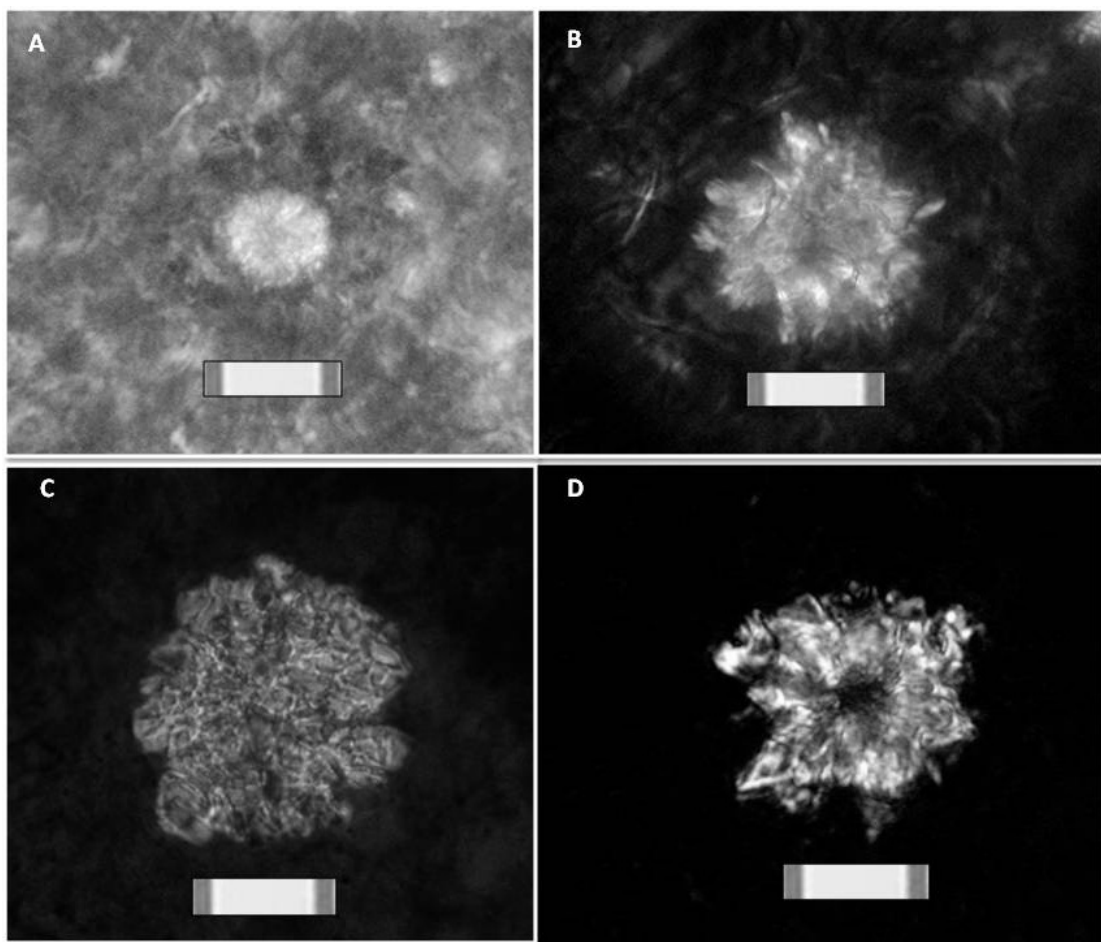

Fig. 3. Biomineralizaciones cálcicas. A: G. lobata. B: G. apiculata. C: G. magellanica. D: G. tinctoria. 
Tabla 1: Abundancia de las drusas. Para cada especie se presenta el mínimo y máximo valor obtenido en cada uno de los 10 conteos realizados.

\begin{tabular}{|lcc|}
\hline \multicolumn{1}{|c}{ Especies } & $\begin{array}{c}\text { Número de drusas por } \\
\text { campo microscópico }\end{array}$ & Clasificación \\
G. lobata & $1-20$ & Escaso \\
G. magellanica & $26-45$ & Abundante \\
G. apiculata & $35-51$ & Abundante \\
G. tinctoria & $170-200$ & Muy abundante \\
\hline
\end{tabular}

Tabla 2: Rango del tamaño de las drusas, observadas en microscopio óptico a $40 x$.

\begin{tabular}{|lc|}
\hline \multicolumn{1}{|c|}{ Especies } & Tamaño de las drusas $(\boldsymbol{\mu m})$ \\
G. lobata & 2,5 a 4,7 \\
G. magellanica & 1,25 a 3 \\
G. apiculata & 1,2 a 5,9 \\
G. tinctoria & 1,25 a 10 \\
\hline
\end{tabular}

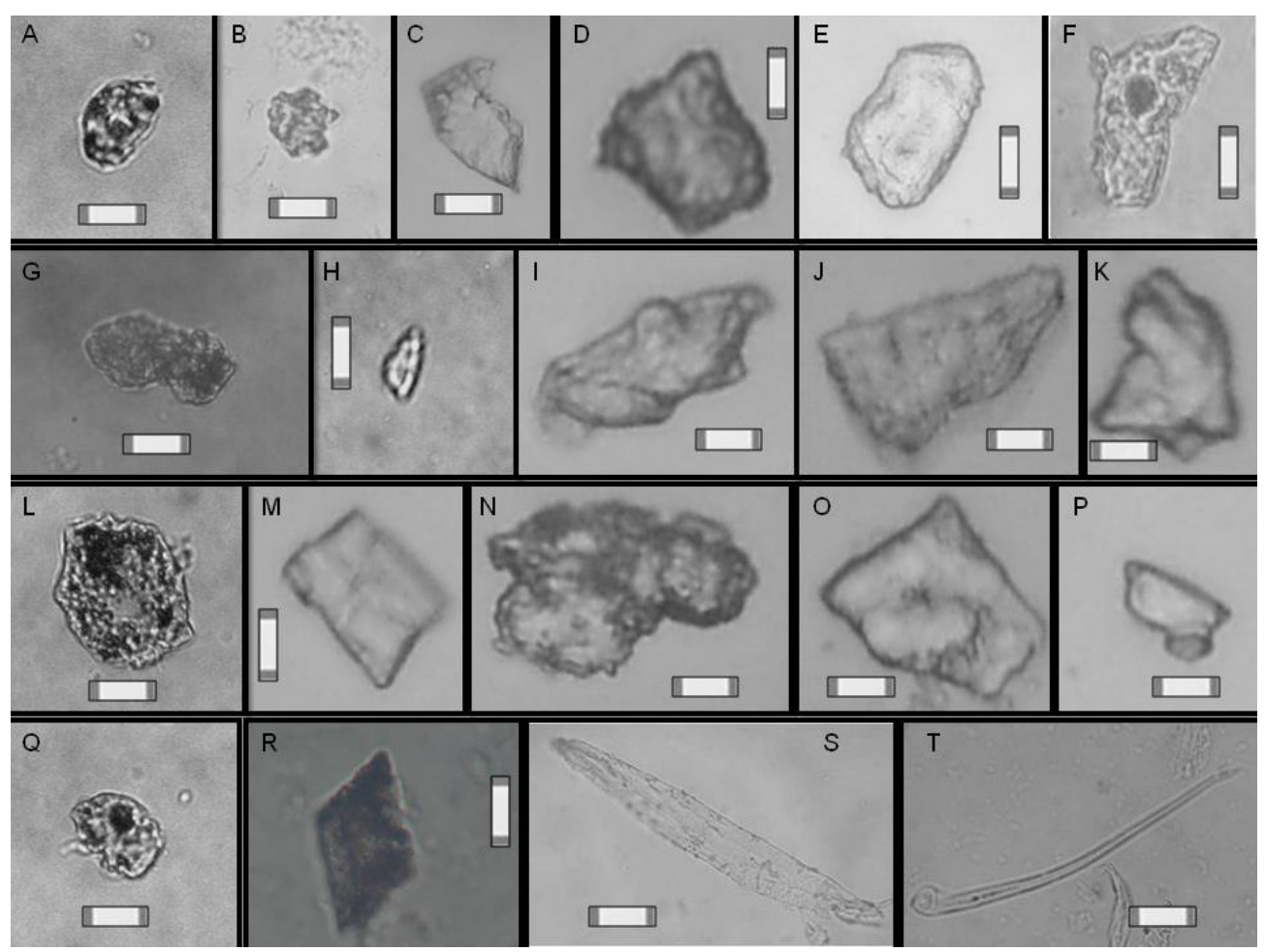

Fig.4. Morfotipos no articulados. Elementos irregulares (Ir). A, B, C, D, E, K, L, N: Elementos irregulares con bordes ondulados. F, J, Q: Elementos irregulares con alguno de sus bordes ondulados y otros bordes rectos. G, H: Elementos irregulares con constricciones bien marcadas, en los que se puede distinguir una parte más ancha y una parte más angosta; Elementos poliédricos ( $\mathrm{Pe}$ ). I, M, R: Elementos poliédricos regulares de bordes lisos. O, P: Elementos poliédricos de bordes lisos con cada uno de sus lados irregulares; Elementos derivados de pelos (Dp). S, T: Pelos uniseriados. Escala gráfica: $10 \mu \mathrm{m}$. 


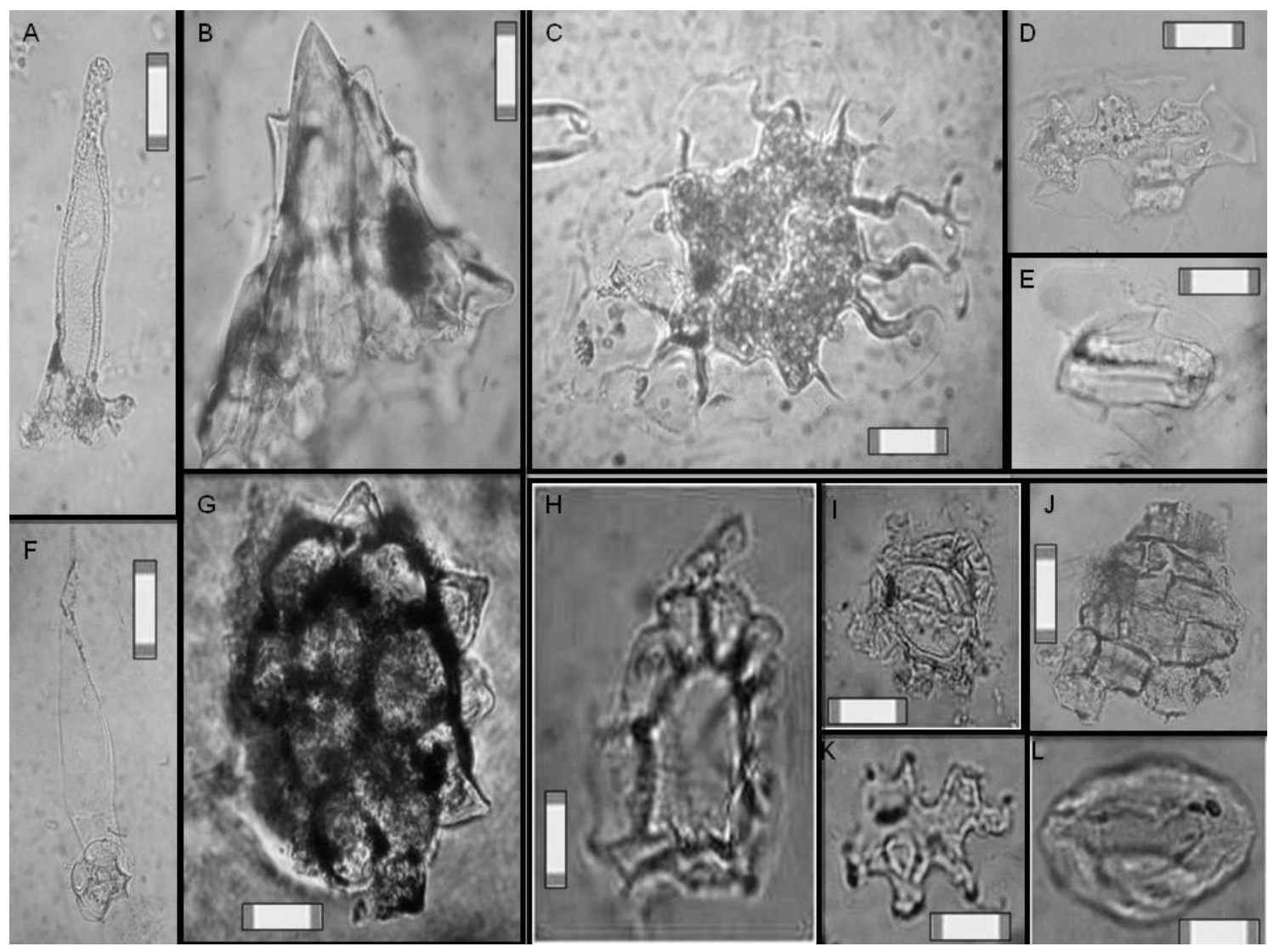

Fig. 5. Biomineralizaciones silíceas de las especies de Gunnera. Morfotipos articulados. A: Pelo unicelular articulado con su base de G. tinctoria. B: Coléter en G. tinctoria. C: Célula epidérmica articulada con pelo de G. apiculata. D: Célula epidérmica articulada con estoma en G. tinctoria. E: Células oclusivas en $G$. tinctoria. F: Pelo unicelular articulado con su base en G. apiculata. G: Coléter en G. apiculata. H: Células de la base del pelo articuladas en G. magellanica. I: Estoma articulado en G. lobata. J: Elemento poliédrico articulado en $G$. apiculata. K: Célula epidérmica de $G$. apiculata. L: Células oclusivas articuladas $G$. magellanica. Escala gráfica: $10 \mu \mathrm{m}$.

Análisis de las asociaciones fitoliticas de las especies

A fin de determinar la importancia de ciertos morfotipos fitolíticos en cada especie y establecer relaciones entre las asociaciones fitolíticas de las mismas, se realizó un análisis de correspondencia considerando las cuantificaciones de los silicofitolitos. Se observó, según los ejes 2 y 3 (31 $\%$ de la variabilidad total), se separa G. apiculata del resto de las especies (Fig. 6), por la abundancia de los morfotipos Ir, de bordes ondulados (Fig. 4A-E).

Al realizar el análisis de correspondencia sólo con las especies patagónicas, agrupadas en el análisis anterior (Fig. 6), se observó, por los ejes 1 y 2 (44\% de la variabilidad total), la separación de las tres especies (Fig. 7). G. lobata se ubicó hacia el eje 1, por los morfotipos irregulares B, F, G, H, I, J; G. magellanica, hacia el eje 2, por la abundancia de los morfotipos C, K, L, M, N, P. mientras que $G$. tinctoria por los morfotipos O, P, Q, R (Fig.4) hacia el eje 1 (Fig. 6). No se incluyó en estén análisis la abundancia de los calciofitolitos, dado que su relevancia está dada por la distribución en la hoja, complementando la información de este análisis. 


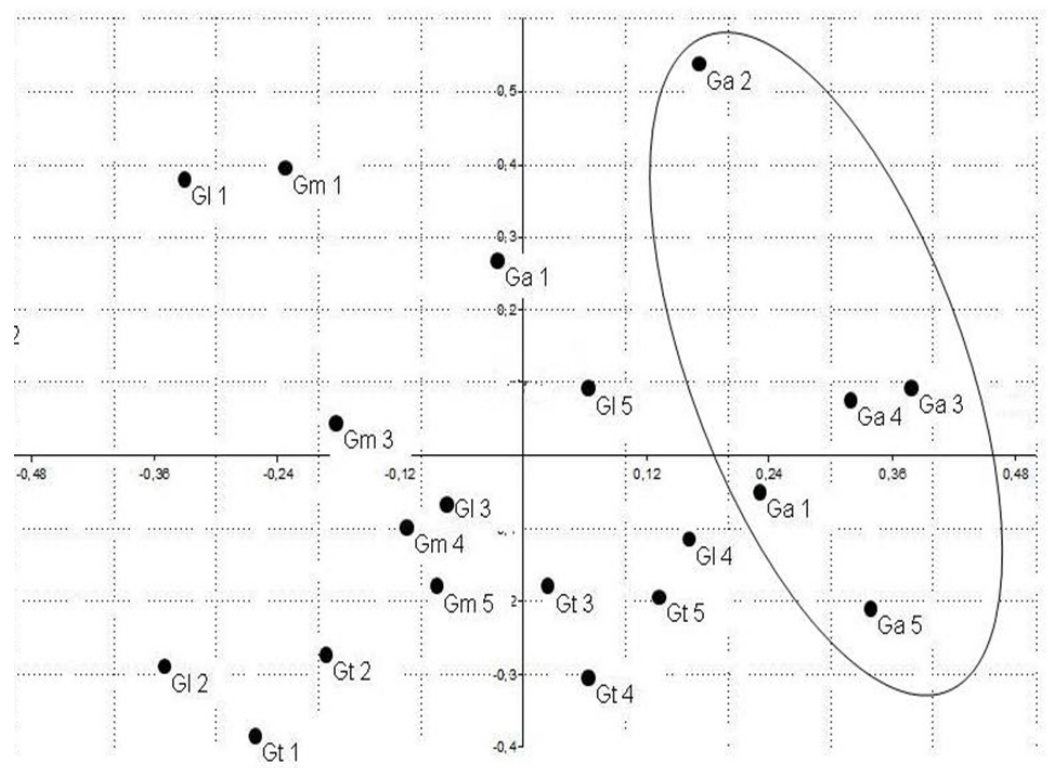

Fig. 6. Resultados del análisis de correspondencia de todos los ejemplares estudiados de G. apiculata (Ga 1, Ga 2, Ga 3, Ga 4, Ga 5), G. lobata (Gl 1, Gl 2, Gl 3, Gl 4, Gl 5), G. magellanica (Gm 1, Gm 2, Gm 3, Gm 4, Gm 5) y G. tinctoria (Gt 1, Gt 2, Gt 3, Gt 4, Gt 5). Se observa una separación de G. apiculata del resto de las especies, mediante el aporte de variabilidad de los morfotipos A, B, C, D y E (Morfotipos sensu Fig.4).

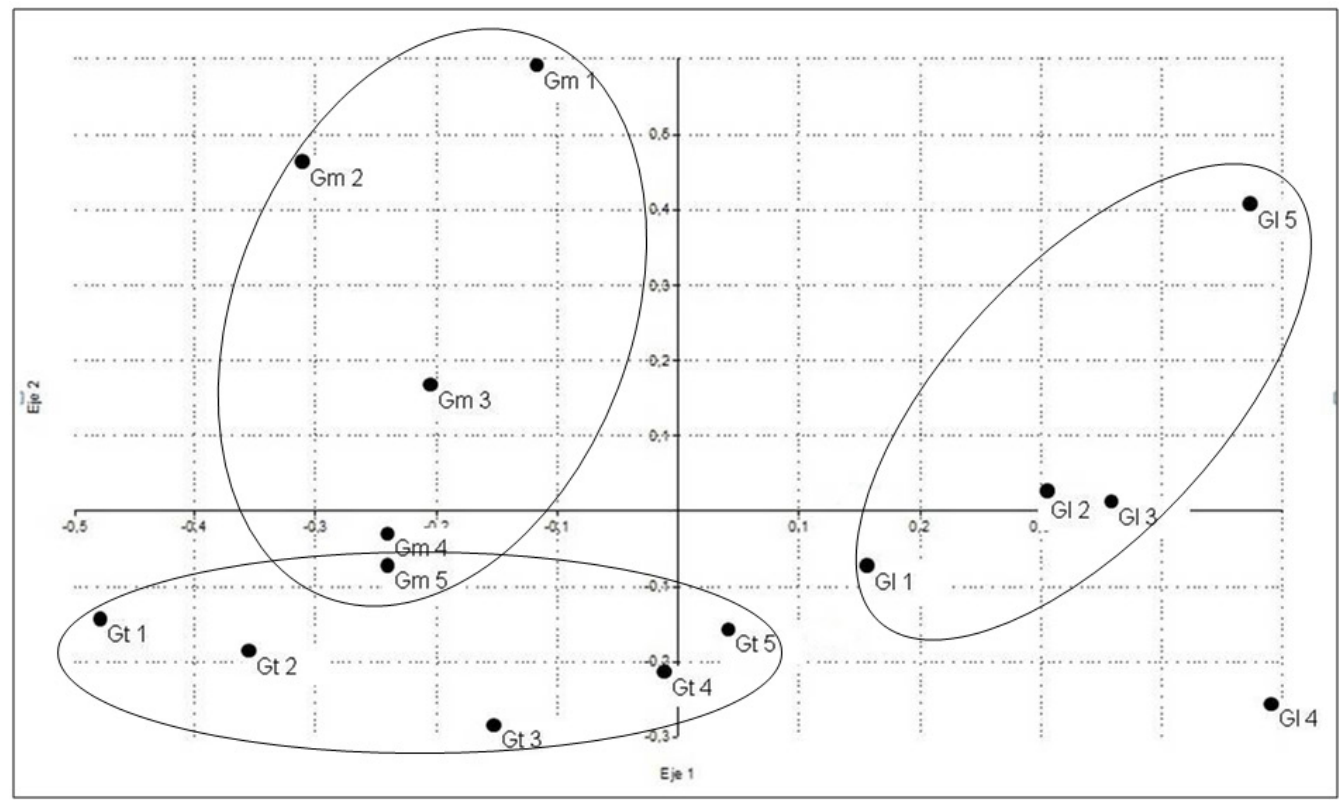

Fig. 7. Análisis de correspondencia de especies patagónicas, Ejemplares estudiados de G. magellanica (Gm 1, Gm 2, Gm 3, Gm, 4, Gm 5), G. tinctoria (Gt 1, Gt 2, Gt 3, Gt 4, Gt 5) y G. lobata (Gl 1, Gl 2, Gl 3, Gl 4, Gl 5), según los ejes (componentes) 1 y 2, por los cuales se diferencian las tres especies por la variabilidad aportada por los morfotipos B, F, G, H, I, J para G. lobata; C, K, L, M, N, P, para G. magellanica; O,P, Q, $\mathrm{R}$ para $G$. tinctoria a estos componentes. 


\section{Discusión}

En este estudio se observó la presencia de cristales de calcio organizados en forma de drusas, tal como Solereder (1908), Metcalfe \& Chalk (1950) y Wilkinson (2000) describen para estas especies. Para G. tinctoria, Wilkinson (2000) menciona la presencia de drusas de calcio en hoja, pero sin especificar sus dimensiones, que en el presente trabajo han sido establecidas en un rango de 1,25-10 $\mu \mathrm{m}$ que las definen como más grandes de las especies estudiadas del género. No se ha encontrado bibliografía sobre la forma y abundancia de las drusas, si bien de acuerdo a estos resultados G. tinctoria resultó la especie que mayor cantidad de drusas posee, las cuales presentan extremos agudos. Para G. magellanica y G. lobata, la presencia de drusas en mesófilo es descripta por Wilkinson (2000) sin hacer mención sobre su abundancia, forma y tamaño. Lo que de acuerdo a nuestros resultados, se puede considerar que en G. lobata las drusas presentan un tamaño mayor al de G. magellanica, sin embargo en esta última son más abundantes. En cuanto al contorno de las drusas en $G$. lobata los extremos de las proyecciones se observan redondeadas, mientras que en G. magellanica truncos. En el presente estudio, se observó en algunas oportunidades, sólo para G. apiculata, la "membrana de Rosanoff" recubriendo los cristales, al igual que lo que describen Rosanoff (1865), Pobeguin (1943), AlRais et al. (1971) Sánchez \& Casabona (1980) y Franceschi \& Nakata (2005).

Las asociaciones silicofitolíticas han sido descriptas por otros autores en estudios previos en especies de Gunnera (Acosta Ricci et al., 2011, 2013; Benvenutto et al., 2011; Fernández Pepi, 2013). El valor taxonómico de las asociaciones fitolíticas fue señalado también en otros trabajos realizados en taxa de nuestra flora, como Festuca (Fernández Pepi et al. 2012b), Stipeae (Arriaga et al. 2009, Zucol, 1996, 1998, 2000), Panicum y Paspalum (Zucol, 1996), algunas especies bonaerenses de Cyperaceae (Fernández Honaine et al. 2009) y Empetrum (Fernández Pepi et al., 2012a). Los morfotipos que se logran identificar para cada una de las especies son herramientas útiles para la separación y diferenciación de las especies estudiadas entre sí.

La concentración de los silicofitolitos en la planta se ve afectada por ciertos factores intrínsecos que pueden relacionarse al taxón estudiado. Para las gramíneas, palmeras y Ciperáceas la producción de fitolitos es muy abundante. En cambio para las dicotiledóneas, la producción es variable según la familia. Algunas familias que suelen tener gran abundancia de sílice y variabilidad de morfotipos son las Urticaceae, Cucurbitaceae, Asteraceae, Magnoliaceae y Moraceae entre otras, cuyos valores varían entre un 1,5-4 \%, aproximadamente (Bertoldi de Pomar, 1975; Hodson et al., 2005; Fernández Pepi, 2013). Por los resultados obtenidos en este estudio, la concentración de sílice está dentro de ese rango de variación, por lo que consideramos que puede incluirse la familia Gunneraceae dentro de este grupo.

La variabilidad en el contenido biomineral entre las especies, puede deberse también, a factores extrínsecos, entre ellos el mecanismo de absorción y depósito de ácido monosilícico en el tejido vegetal. En modo directo a su vez, la disponibilidad de dicho ácido en el suelo se relaciona con el $\mathrm{pH}$ del mismo.

Por otro lado la temperatura y humedad del ambiente quienes incrementan la tasa de transpiración y depósito de sílice en la planta (Bertoldi de Pomar, 1975). En este trabajo, no puede quedar descartada totalmente la posibilidad de que la variabilidad en el contenido de sílice de las especies, sea resultado de las variables ambientales bajo las cuales se desarrollan, dado que cada región fitogeográfica en las que las especies habitan posee diferencias notorias en sus características ambientales. Gunnera apiculata se desarrolla en la selva de las yungas donde las temperaturas alcanzan $\operatorname{los} 27^{\circ} \mathrm{C}$ y las lluvias estivales son comunes en los meses de diciembre a marzo (Cabrera, 1971). Razones por las cuales se puede pensar que las tasas de transpiración en las especies del sotobosque y no solo en G. apiculata son elevadas. El porcentaje de biomineralización silícea se relaciona en forma directa con la tasa de transpiración, por lo que se explicaría de esta forma como G. apiculata adquiere un índice de biomineralización silícea muy superior al de las demás especies. G. lobata, G. magellanica y $G$. tinctoria, distribuidas en la Patagonia argentina se encuentran bajo un clima húmedo, de frio a cálido dependiendo de la época del año, si bien no supera 
$\operatorname{los} 20^{\circ} \mathrm{C}$ (Cabrera, 1971). Lo que permite estimar que en estas especies la tasa de transpiración e incorporación de sílice es menor, resultando en un menor índice de biomineralización silícea.

\section{Conclusiones}

Para cada una de las especies los índices de biomineralización total obtenidos, permitieron establecer diferencias significativas entre G. lobata y las restantes especies. Esta especie presenta menor contenido biomineral es G. lobata, por ende al analizar el contenido biomineral silíceo y cálcico también adquiere valores bajos en comparación con las especies restantes. Por otro lado, G. tinctoria, presenta el mayor contenido biomineral total y por ende, silíceo y cálcico también. Los morfotipos silíceos presentes en el material estudiado y descripto permiten separar y diferenciar a las especies entre sí. La forma del extremo de los ejes de las drusas es un carácter que determina diferencias entre las especies del género. El nuevo enfoque biomineral aportó nuevas características de diferenciación intraespecíifica, El conocer el contenido y las asociaciones de cada tipo de biomineralización permite elaborar una herramienta de análisis para estudios ambientales en diferentes regiones donde habita cada especie, a partir de la cuantificación de estos microrestos, en especial los silicofitolitos, que toleran sin alterarse, una variación de $\mathrm{pH}$ del suelo. El nuevo enfoque del estudio sistemático de un grupo de vegetales mediante las características de sus depósito biomeneral aportó nuevas pautas de diferenciación intraespecífica, dándole así un valor taxonómico relevante a a estas acumulaciones y las asociaciones fitolíticas características.

\section{Agradecimientos}

A la Dra. C. Ezcurra (INIBIOMA-CONICET), a los Licenciados Eva Bulacio, Hugo Ayarde y Sebastián Buedo (LIL), a la Dra. A.S. Moretto, Lic. J.M. Escobar (CADIC-CONICET) y Lic. E.C. Alvarenga (MACNCONICET) por la colección y envío de materiales frescos. A los Curadores de los Herbarios consultados: Dra. N. Deginani (SI), Lic. L. Iharlegui (LP) y Sra. D. Monetero (BA), por las facilidades otorgadas. El trabajo forma parte del seminario de tesis de grado de la primera autora, Universidad CAECE.

\section{Bibliografía}

ACOSTA RICCI, Y.C., ARRIAGA, M.O. \& FERNÁNDEZ PEPI, M.G. 2011. Descripción y cuantificación biomineral de Gunnera magellanica Lam. (Gunneraceae). Bol. Soc. Argent. Bot. 46 (Supl): 80.

ACOSTA RICCI, Y. C., FERNÁNDEZ PEPI, M.G \& ARRIAGA, M. O. 2013. Comparación de la composición biomineral de cuatro especies del género Gunnera. Bol. Soc. Argent. Bot. 48 (Supl): 31.

AGUIRRE, C., CHÁVEZ, T., GARCÍA, P. \& RAYA, P. 2007. El silicio en los organismos vivos. Interciencia 32: 504-509.

AL-RAIS, A.H., MYERS, A. \& WATSON, L. 1971.The isolation and proprieties of oxalate crystals from plants. Ann. Bot. 35: 1213-1218.

ARRIAGA, M. O., ZUCOL, A. F., FERNÁNDEZ PEPI, M. G. \& PATERER, N. I. 2009. Análisis fitolítico comparado de las especies sudamericanas de Stipeae (Poaceae) como una herramienta taxonómica. Bol. Soc. Argent. Bot. 44 (Supl): 140.

BENVENUTO, L., FERNÁNDEZ HONAINE, M., OSTERRIETH, M., CORONATO, A. \& RABASSA, J. 2011. Silicophytoliths in Holocene peatlands and fossil peat layers from Tierra del Fuego, Argentina, southernmost South America. Quater. Internat. 30: $1-14$.

BERTOLDI DE POMAR, H. 1975. Los silicofitolitos: sinopsis de su conocimiento. Darwiniana 19: 173206.

BORBA-ROSCHEL, M., ALEXANDRE, A., VARAJÃO AFD., MEUNIER, J.D., VARAJÃO, C.A.C. \& COLIN, F. 2006. Phytoliths as indicators of pedogenesis and paleoenvironmental changes in the Brazilian cerrado. J. Geochem. Explor. 88: 172-176.

CABRERA, A.L. 1971. Fitogeografía de la República Argentina. Bol. Soc. Argent. Bot. 14: 1-42.

D’ AMBROGIO DE ARGÜESO, A. 1986. Manual de técnicas de Histología Vegetal. Hemisferio Sur, Buenos Aires.

FERNÁNDEZ HONAINE, M., OSTERRIETH, M.L. \& ZUCOL, A.F. 2009. Phytolith analysis of Cyperaceae from the Pampean region, Argentina. Austr. J. Bot. 57: 1-11.

FERNÁNDEZ PEPI, M.G. 2013. Estudios fitoliticos de las comunidades vegetales del ecotono fueguino como una herramienta para reconocer sus variaciones de composición en el pasado reciente. Tesis de Doctorado en Ciencias Biológicas. Facultad de Ciencias Exactas y Naturales, UBA.

FERNÁNDEZ PEPI, M. G., ARRIAGA, M. O. \& ZUCOL, A. F. 2012a. Leaf anatomy and biomineralization in Empetrum rubum Valh ex Willd. (Ericaceae). Botánica Complut. 36: 113-121. 
FERNÁNDEZ PEPI, M. G., ZUCOL, A. F. \& ARRIAGA, M. O. 2012b. Comparative phytolith analysis of Festuca (Pooidae: Poaceae) native species of Tierra del Fuego, Argentina. Botany 90: 1113-1124.

FRANCESCHI, V. R. \& NAKATA, P. A. 2005. Calcium oxalate in plants: formation and function. Annu. Rev. Plant Biol. 56: 41-71.

HAMMER, O., HARPER, D. A. T. \& RYAN, P. D. 2007. PAST-PAlaeontological STatistics 1. 75. http://folk. uio.no/ohammer/past.

HODSON, M. J., WHITE, P. J., MEAD, A. \& BROADLEY, M. R. 2005. Phylogenetic variation in the silicon composition in plants. Ann. Bot. 96: 1027-1046.

LABOURIAU, L. G. 1983. Phytolith work in Brazil, A minireview. The phytolitarien Newsl. 2 (2): 6-11.

LERSTEN, N. R. \& HORNER, H. T. 2009. Crystal diversity and macropatterns in leaves of Oleaceae. Plant Syst. Evol. 282: 87-102.

METCALFE, C.R. \& CHALK, L. 1950. Anatomy of the Dicotyledons. Leaves, stem, and wood in relation to taxonomy with notes on economic uses. Vol. I: Clarendon Press, Oxford.

MOLINA, AM. 1978. El género Gunnera en Argentina y el Uruguay (Gunneraceae). Darwiniana 21: 473 489).

NAPP ZINN, K. 1984. Anatomie des Blattes. II. Blattanatomie der Angiospermen. B. Experimentelle und okologische anatomie des Angiospermenblattes 1: 1028-49.

PATTERER, N. I., PASSEGGi, E. \& ZUCOL, A. F. 2011. Análisis fitolíticos de los suelos del sudoeste de la provincia de Entre Ríos (Argentina) como una herramienta para comprender sus procesos pedológicos. Revista Mex. Cs. Geol. 28: 132-146.

PÉREZ CUADRA, V. \& HERMANN, P. 2013. Characterization and macropattern of calcicum oxalate phytoliths in Argentinean endemic species of Chenopodioideae (Amaranthaceae). Quarter. Internat. 283: 83-88.

POBEGUIN, T. 1943. Les oxalates de calcium chez quelques Angiosperms. Étude physicochimique, formation, destin. Ann. Sci. Nat. Bot. II Sér. 4:1-95.

ROSANOFF, S. 1865. Uber die Kystalldrusen im Marke von Kerria japonica und Ricinus communis. Bot. Zeit. 23: 329-330.

SÁNCHEZ, E. \& CASABONA, M. I. 1980. Presencia de cristales en el gineceo de las Gramineae. Physis (Buenos Aires), Secc. C 39 (96): 15-20.
SOLEREDER, H. 1908. Solereder's anatomy of the dicotyledons. Vol. I. Claredon Press, Oxford.

DEY, S.B., GHOSH, R., SHEKHAR, M., MUKHERJEE, B. \& BERA, S. 2015. What drives elevational pattern of phytolith diversity in Thysanolaena maxima (Roxb.) O. Ktze? A study from the Darjeeling Himalayas. Flora 211: 51-61.

WEBB, M.A. 1999. Cell-mediated crystallization of calcium oxalate in plants. Plant Cell 11: 751-761.

WILKINSON, H.P. 2000. A revision of the anatomy of Gunneraceae. Bot. J. Linn. Soc. 134: 233-226.

ZUCOL, A. F. 1996. Microfitolitos de las Poaceae argentinas: I. Microfitolitos foliares de algunas especies del género Stipa (Stipeae: Arundinoideae: Poaceae), en la provincia de Entre Ríos. Darwiniana 34: 151-172.

ZUCOL, A. F. 1998. Microfitolitos de la Poaceae argentinas: II. Microfitolitos foliares de algunas especies del género Panicum (Paniceae: Panicoideae: Poaceae), en la provincia de Entre Ríos. Darwiniana 36: 29-50.

ZUCOL, A. F. 2000. Fitolitos de Poaceae argentinas: III. Fitolitos foliares de algunas especies del género Paspalum (Paniceae: Panicoideae: Poaceae), en la provincia de Entre Ríos. Darwiniana 38: 11-32.

ZUCOL, A. F., BREA, M. \& BELLOSI, E. S. 2010. Phytolith studies in Gran Barranca (central Patagonia, Argentina): The middle-late Eocene. In: MADDEN, R.H., CARLINI, A. A., VUCETICH, M. G. \& Kay, R.F. (eds.), The Paleontology of Gran Barranca. Evolution and Environmental Change through the Middle Cenozoic of Patagonia, pp. 317 340. Cambridge University Press, New York.

ZULOAGA, F. O. \& MORRONE, O. 1999. Catálogo de las Plantas Vasculares de la Argentina II. Monogr. Syst. Bot. Missouri Bot. Gard 74: 1-1269.

ZULOAGA, F.O., MORRONE, O. \& BELGRANO, M. (Eds.). 2008. Catálogo de Plantas Vasculares del Cono Sur (Argentina, sur de Brasil, Chile, Paraguay y Uruguay). I. Monogr. Syst. Bot. Missouri Bot. Gard. 107:1-983.

Recibido el 08 de mayo de 2015, aceptado el 06 de agosto de 2015. 